Research Paper

\title{
Treatment Strategies and Prognostic Factors of Primary Gastric Diffuse Large B Cell Lymphoma: A Retrospective Multicenter Study of 272 Cases from the China Lymphoma Patient Registry
}

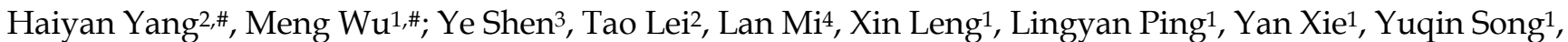
Xinan Cen ${ }^{3 凶 *}$, Jun Zhu ${ }^{1 凶 *}$

1. Key laboratory of Carcinogenesis and Translational Research (Ministry of Education), Department of Lymphoma, Peking University Cancer Hospital \& Institute, Postal address: No 52, Fucheng Road, Haidian District, Beijing, China. 100142

2. Department of Lymphoma, Zhejiang Cancer Hospital, Postal address: No. 1, Bansan Road, Hangzhou, Zhejiang, China

3. Department of Hematology, Peking University First Hospital, Postal address: No. 8, Xishiku Street, Xicheng District, Beijing, China. 100034

4. Key laboratory of Carcinogenesis and Translational Research (Ministry of Education), Department of outreach and industrial Affairs, Peking University Cancer Hospital \& Institute, Postal address: No 52, Fucheng Road, Haidian District, Beijing, China. 100142

\# Haiyan Yang and Meng Wu contributed equally to this manuscript.

* Xinan Cen and Jun Zhu jointly supervised this work.

$\triangle$ Corresponding authors: Xinan Cen: E-mail address: xinancen@hotmail.com Tel: +86-13501385729. Jun Zhu: E-mail address: zhu-jun2017@outlook.com; Tel: +86-010-8819-6109; Fax: +86-010-8819-6115

(C) Ivyspring International Publisher. This is an open access article distributed under the terms of the Creative Commons Attribution (CC BY-NC) license (https://creativecommons.org/licenses/by-nc/4.0/). See http://ivyspring.com/terms for full terms and conditions.

Received: 2019.02.17; Accepted: 2019.05.08; Published: 2019.06.10

\begin{abstract}
Background: The respective and combinatorial roles of surgery, Rituximab and chemotherapy in primary gastric diffuse large $B$ cell lymphoma (PGDLBCL) therapy remained unclear. The purpose of the study was to evaluate present treatment strategies and prognostic factors of PGDLBCL.

Methods: 272 cases (from 1994-1 to 2015-12) were retrospectively analyzed. According to the therapy regimen, patients were classified into four groups: chemotherapy $(C)$, chemotherapy + surgery $(C+S)$, Rituximab + chemotherapy $(R+C)$, and Rituximab + chemotherapy + surgery $(R+C+S)$.

Results: The 3-year progression-free survival (PFS) and 3-year overall survivals (OS) of the entire cohort were $77.0 \%$ and $81.2 \%$ respectively (median follow-up time: 44.3 months). Survival of surgery-treated patients was superior to the survival of those receiving drug therapy alone (PFS: $82.6 \%$ vs. $74.7 \%, p=0.015$; OS: $87.8 \%$ vs. $78.6 \%, p=0.036)$. Rituximab showed significant clinical benefit in OS (87.1\% vs. $75.0 \%, p=0.007)$, especially in advanced-stage or high risk (IPI 3-5) patients. Group C had the lowest PFS and OS among the four groups, while the survival of other three groups were similar (Group $C$ vs. Group $C+S$ vs. Group $R+C$ vs. Group $R+C+S$ : 3 -year PFS: $67.2 \%$ vs. $81.4 \%$ vs. $81.2 \%$ vs. $81.8 \%, p=0.002$; 3 -year OS: $68.4 \%$ vs. $85.4 \%$ vs. $87.2 \%$ vs. $88.6 \%$, $p<0.001)$. Multivariate analysis showed that IPI and therapy regimens were highly predictive for both PFS and OS.

Conclusions: Our results suggested that the combinations of chemotherapy and surgery, or chemotherapy and Rituximab, are superior to other treatment strategies for PGDLBCL. IPI and therapy regimens are independent predictors of outcomes. Future prospective trial is warranted.
\end{abstract}

Key words: Primary gastric diffuse large B cell lymphoma (PGDLBCL), Treatment strategies, Prognostic factors, Retrospective multicenter analysis

\section{Introduction}

Diffuse large B cell lymphoma (DLBCL) is the most common NHL subtype, accounting for approximately $32.5 \%$ of all NHL diagnosed annually.[1] Among all DLBCL patients, 32\% presents with extranodal primary sites, in which gastrointestinal tract $(34 \%)$ is the most common 
primary site.[2] In the primary gastrointestinal lymphoma, the most commonly affected site is stomach (approximately 50-60\%), followed by small intestines (30\%) and large intestines (about 10\%).[3]

Primary gastric lymphoma (PGL), following adenocarcinoma,[4] ranked as second most common gastric malignant tumor (2-8\%).[5] DLBCL is the main histological subtype of PGL, accounting for $38-59 \%$.[3, 6-8] Gastrectomy used to be the first-line therapy in primary gastric DLBCL (PGDLBCL) two decade ago. However, considering the efficacy and quality of life, more and more non-surgical therapies, such as chemotherapy and radiotherapy, have been used in PGDLBCL patients, especially in the Rituximab era. The optimal treatment for PGDLBCL still remains controversial. We thus conducted a retrospective analysis of 272 cases to evaluate present treatment strategies and prognostic factors of PGDLBCL.

The China Lymphoma Patient Registry included the following five Chinese institutions: Peking University Cancer Hospital \& Institute, Peking University First Hospital, Zhejiang Cancer Hospital, Tianjin Medical University Cancer Institute and Hospital, and Harbin Medical University Cancer Hospital, the former three institutions provided the data of PGDLBCL patients for this study.

\section{Patients and Methods}

\section{Patients and therapy regimens}

A total of 303 patients with previously untreated PGDLBCL at three centers of CLAP were reviewed between January 1994 and December 2015. Their histopathologic data were identified by the pathology departments according to "the 2008 WHO classification of tumors of hematopoietic and lymphoid tissues".[9] Imaging and endoscopic examination were used together to define stage and evaluate therapy response. Stage was defined according to the Lugano Staging System for Gastrointestinal Lymphomas.[10] Response criteria were defined according to the International Working Group Recommendation.[11] The analysis was approved by the Medical Ethics Committee of Peking University Cancer Hospital \& Institute.

Twenty-four patients did not receive consecutive therapy from each institution, and were excluded from analysis. Among the 279 patients, two patients died as a result of gastrointestinal bleeding before any anti-lymphoma therapy could be administered. Five patients received palliative surgery only (The only goal of palliative surgery was to alleviate obstruction), four of which died as a result of progressive disease within three months after surgery, the fifth patient did not receive other anti-lymphoma therapy after surgery, who was alive as the last follow-up reported
(OS 155.9 months). Excepted the seven patients above, the rest 272 patients received either chemotherapy (193 cases) or the combination of chemotherapy and surgery (79 cases). In order to evaluate present treatment strategies, the 272 patients were retrospectively analyzed at last. According to the therapy regimen, patients were further classified into four groups: chemotherapy (C, 80 cases), chemotherapy + surgery $(\mathrm{C}+\mathrm{S}, 50$ cases $)$, Rituximab + chemotherapy $(\mathrm{R}+\mathrm{C}, 112$ cases), and Rituximab + chemotherapy + surgery $(\mathrm{R}+\mathrm{C}+\mathrm{S}, 30$ cases $)$. All patients received $\mathrm{CHOP}$-like (the main drugs in CHOP-like regimen was cyclophosphamide + doxorubicin + vincristine \pm prednisone) as chemotherapy regimen. The combination of chemotherapy and surgery meant that the patients received a complete resection of gastric tumor first followed by chemotherapy as described above. Radiotherapy was used as palliative therapy only in this study (24 cases).

\section{Statistic methods}

All patients were followed up from inpatients, outpatients or telephone. Follow up time was defined from the date of diagnosis until death or the last follow-up. Overall survival (OS) was calculated from the date of diagnosis until death as a result of any cause. Progression-free survival (PFS) was measured from the date of diagnosis to the first relapse, progressive disease, or last follow-up. OS and PFS rates were estimated using the Kaplan-Meier method. To identify prognostic variables for OS and PFS, univariate analysis was performed for the baseline clinical features, therapy regimens, and complications. Log-rank tests and Cox regression models were used to analyze the univariate and multivariate impacts of various prognostic factors. All data was analyzed with IBM SPSS Statistics, version 22.0.

\section{Results}

\section{Clinical features}

The median age for the 272 patients of PGDLBCL was 56 years (range: 16-87 years), and the male-to-female ratio was $0.97: 1$. More than half of patients $(153 / 270)$ presented with local-stage PGDLBCL. The patients with $0-1$ score of International Prognostic Index (IPI) were accounted for $55.1 \%(146 / 265)$. The rest showed either 2 score $(20.8 \%, 55 / 265)$, or 3 score $(16.2 \%, 43 / 265)$, or $4-5$ score $(7.9 \%, 21 / 265)$. Other findings included B symptom $46.1 \%$ (125/269), anemia $48.5 \%$ (132/272), gastrointestinal bleeding before treatment 31.5\% (69/219), elevated Lactate dehydrogenase (LDH) 29.5\% (78/264), elevated Erythrocyte Sedimentation Rate (ESR) $62.6 \%$ (109/174). The most common lesion 
sites were the body $(43.9 \%, 115 / 262)$ or antrum $(40.1 \%, 105 / 262)$ of the stomach. Patient baseline clinical features were listed in Table 1.

\section{Survival in different therapy regimens}

With a median follow-up time of 44.3 months (range: 0.2-233.1), the 3-year PFS and 3-year OS of the entire cohort were $77.0 \%$ and $81.2 \%$ respectively (Figure 1). In the entire cohort, surgery-treated patients were superior to those receiving drug therapy alone in both PFS and OS (3-year PFS: 82.6\% vs. $74.7 \%, p=0.015$; 3 -year OS: $87.8 \%$ vs. $78.6 \%, p=0.036$; Figure 2). Rituximab showed significant clinical benefit in OS $(87.3 \%$ vs. $79.3 \%, p=0.09)$. Patients receiving Rituximab had higher PFS, although without statistical significance $(81.0 \%$ vs. $77.0 \%$, $p=0.063$; Figure 3 ). The inclusion of radiotherapy during initial treatment (24 cases) in this cohort did not improve PFS $(56.5 \%$ vs. $79.3 \%, p=0.012)$ or OS (73.4\% vs. $82.9 \%, p=0.178)$.

In the local-stage (Stage I and Stage II) cohort, the advantage of surgery was not significant in either PFS or OS (3-year PFS: $91.4 \%$ vs. $90.2 \%, p=0.163$; 3-year OS: 95.7\% vs. 91.2\%, $p=0.088)$. Patients received Rituximab did not show any statistically significant improvement compared to those who did not receive Rituximab (3-year PFS: 93.3\% vs. 88.0\%, $p=0.128$; 3-year OS: $95.9 \%$ vs. $89.4 \%, p=0.074)$. In advanced-stage (Stage IV) cohort, Rituximab showed significant clinical benefit in OS (3-year PFS: $66.9 \%$ vs. $54.2 \%, p=0.210$; 3-year OS: $75.9 \%$ vs. $57.5 \%, p=0.036$ ). The PFS and OS of patients after surgery were relative higher, even without statistically significance (3-year PFS: $67.2 \%$ vs. $57.7 \%, p=0.103$; 3-year OS: $74.5 \%$ vs. $64.6 \%, p=0.281)$.

Table 1. Patient Clinical Features in Different Therapy Groups

\begin{tabular}{|c|c|c|c|c|c|}
\hline & $\operatorname{ALL}(\mathrm{N}=272)$ & $\begin{array}{l}\text { Chemotherapy } \\
(\mathrm{N}=80)\end{array}$ & $\begin{array}{l}\text { Chemotherapy+Surgery } \\
(\mathrm{N}=50)\end{array}$ & $\begin{array}{l}\text { Rituximab+Chemotherapy } \\
(\mathrm{N}=112)\end{array}$ & $\begin{array}{l}\text { Rituximab+Chemotherapy+ } \\
\text { Surgery }(\mathrm{N}=30)\end{array}$ \\
\hline & $\mathrm{N}(\%)$ & $\mathrm{N}(\%)$ & $\mathrm{N}(\%)$ & $\mathrm{N}(\%)$ & $\mathrm{N}(\%)$ \\
\hline \multicolumn{6}{|l|}{ Sex } \\
\hline Male & $134(49.3 \%)$ & $42(52.5 \%)$ & $28(56.0 \%)$ & $50(44.6 \%)$ & $14(46.7 \%)$ \\
\hline Female & $138(50.7 \%)$ & $38(47.5 \%)$ & $22(44.0 \%)$ & $62(55.4 \%)$ & $16(53.3 \%)$ \\
\hline \multicolumn{6}{|l|}{ Age } \\
\hline$\leq 60$-year-old & $169(\%)$ & $48(60.0 \%)$ & $34(68.0 \%)$ & $68(60.7 \%)$ & $19(63.3 \%)$ \\
\hline$>60$-year-old & $103(\%)$ & $32(40.0 \%)$ & $16(32.0 \%)$ & $44(39.3 \%)$ & $11(36.7 \%)$ \\
\hline \multicolumn{6}{|l|}{ Lugano Stage } \\
\hline Stage I & $61(22.6 \%)$ & $11(13.9 \%)$ & $14(28.6 \%)$ & $28(25.0 \%)$ & $8(26.7 \%)$ \\
\hline Stage II & $92(34.1 \%)$ & $25(31.6 \%)$ & $21(42.9 \%)$ & $39(34.8 \%)$ & $7(23.3 \%)$ \\
\hline Stage IV & $117(43.3 \%)$ & $43(54.4 \%)$ & $14(28.6 \%)$ & $45(40.2 \%)$ & $15(50.0 \%)$ \\
\hline Unknown & 2 & 1 & 1 & - & - \\
\hline \multicolumn{6}{|l|}{ B Symptom } \\
\hline No & $146(53.9 \%)$ & $40(50 \%)$ & $27(55.1 \%)$ & $59(52.7 \%)$ & $20(66.7 \%)$ \\
\hline Yes & $125(46.1 \%)$ & $40(50 \%)$ & $22(44.9 \%)$ & $53(47.3 \%)$ & $10(33.3 \%)$ \\
\hline Unknown & 1 & - & 1 & - & - \\
\hline \multicolumn{6}{|l|}{ ECOG PS } \\
\hline $0-1$ & $255(94.8 \%)$ & $77(97.5 \%)$ & $45(91.8 \%)$ & $106(95.5 \%)$ & $27(90.0 \%)$ \\
\hline$>1$ & $14(5.2 \%)$ & $2(2.5 \%)$ & $4(8.2 \%)$ & $5(4.5 \%)$ & $3(10.0 \%)$ \\
\hline Unknown & 3 & 1 & 1 & 1 & - \\
\hline \multicolumn{6}{|l|}{$\mathrm{LDH}$} \\
\hline $\operatorname{Normal}(\leq 240 \mathrm{U} / \mathrm{L})$ & $186(70.5 \%)$ & $52(65.8 \%)$ & $34(70.8 \%)$ & $77(71.3 \%)$ & $23(79.3 \%)$ \\
\hline Elevated (>240U/L) & $78(29.5 \%)$ & $27(34.2 \%)$ & $14(29.2 \%)$ & $31(28.7 \%)$ & $6(20.7 \%)$ \\
\hline Unknown & 8 & 1 & 2 & 4 & 1 \\
\hline \multicolumn{6}{|l|}{ IPI } \\
\hline $0-1$ & $146(55.1 \%)$ & $39(49.4 \%)$ & $29(63.0 \%)$ & $63(56.8 \%)$ & $15(51.7 \%)$ \\
\hline 2 & $55(20.8 \%)$ & $22(27.8 \%)$ & $9(19.6 \%)$ & $20(18.0 \%)$ & $4(13.8 \%)$ \\
\hline 3 & $43(16.2 \%)$ & $14(17.7 \%)$ & $4(8.7 \%)$ & $19(17.1 \%)$ & $6(16.2 \%)$ \\
\hline $4-5$ & $21(7.9 \%)$ & $4(5.1 \%)$ & $4(8.7 \%)$ & $9(8.1 \%)$ & $4(7.9 \%)$ \\
\hline Unknown & 7 & 1 & 4 & 1 & 1 \\
\hline \multicolumn{6}{|l|}{ HGB } \\
\hline$\geq 120 \mathrm{~g} / \mathrm{L}$ (Male) or $\geq 110 \mathrm{~g} / \mathrm{L}$ (Female) & $140(51.5 \%)$ & $43(53.8 \%)$ & $20(40.0 \%)$ & $62(55.4 \%)$ & $15(50.0 \%)$ \\
\hline $90 \leq \mathrm{HGB}<120 \mathrm{~g} / \mathrm{L}$ (Male) or & $79(29 \%)$ & $20(25.9 \%)$ & $22(44.0 \%)$ & $28(25.0 \%)$ & $9(30.0 \%)$ \\
\hline \multicolumn{6}{|l|}{$90 \leq \mathrm{HGB}<110 \mathrm{~g} / \mathrm{L}$ (Female) } \\
\hline$<90 \mathrm{~g} / \mathrm{L}$ & $53(19.5 \%)$ & $17(21.3 \%)$ & $8(16.0 \%)$ & $22(9.6 \%)$ & $6(20.0 \%)$ \\
\hline \multicolumn{6}{|l|}{ Gastrointestinal bleeding before treatment } \\
\hline No & $150(68.5 \%)$ & $45(69.2 \%)$ & $21(70.0 \%)$ & $69(69.0 \%)$ & $15(62.5 \%)$ \\
\hline Yes & $69(31.5 \%)$ & $20(30.8 \%)$ & $9(30.0 \%)$ & $31(31.0 \%)$ & $9(37.5 \%)$ \\
\hline Unknown & 53 & 15 & 20 & 12 & 6 \\
\hline \multicolumn{6}{|l|}{ ESR } \\
\hline Normal $(\leq 15 \mathrm{~mm} / \mathrm{h})$ & $65(37.4 \%)$ & $20(33.3 \%)$ & $4(22.2 \%)$ & $36(45.0 \%)$ & $5(31.3 \%)$ \\
\hline Elevated $(>15 \mathrm{~mm} / \mathrm{h})$ & $109(62.6 \%)$ & $40(66.7 \%)$ & $14(77.8 \%)$ & $44(55.0 \%)$ & $11(68.8 \%)$ \\
\hline Unknown & 98 & 20 & 32 & 32 & 14 \\
\hline
\end{tabular}

ECOG PS: Eastern Cooperative Oncology Group performance status; LDH: Lactate dehydrogenase; IPI: International Prognostic Index; HGB, Hemoglobin; ESR: Erythrocyte sedimentation rate. 


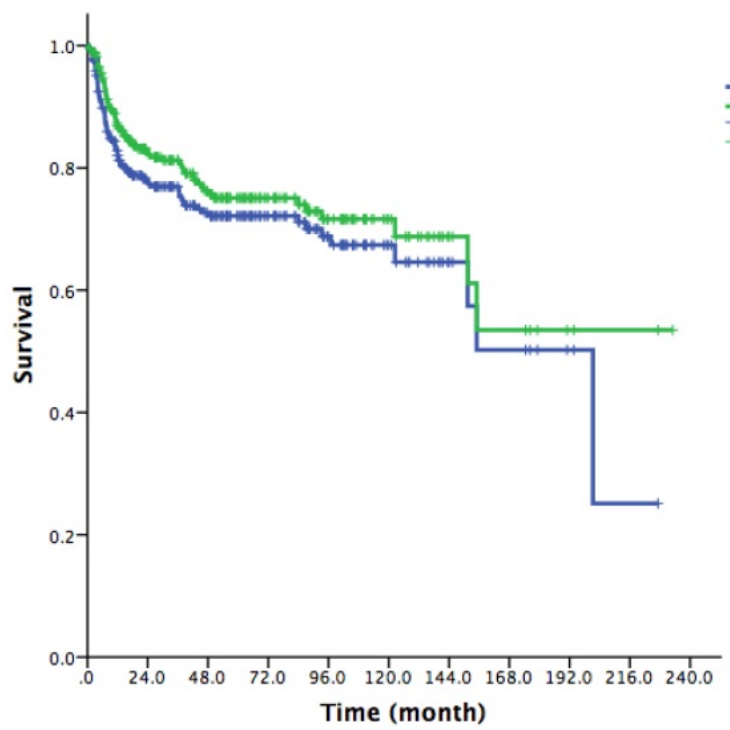

All patients
$(n=272)$

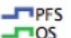

+ PFS-censored
+ OS-censored

Time (month)

Figure 1. 272 patients Kaplan-Meier curve of overall survival and progression free survival
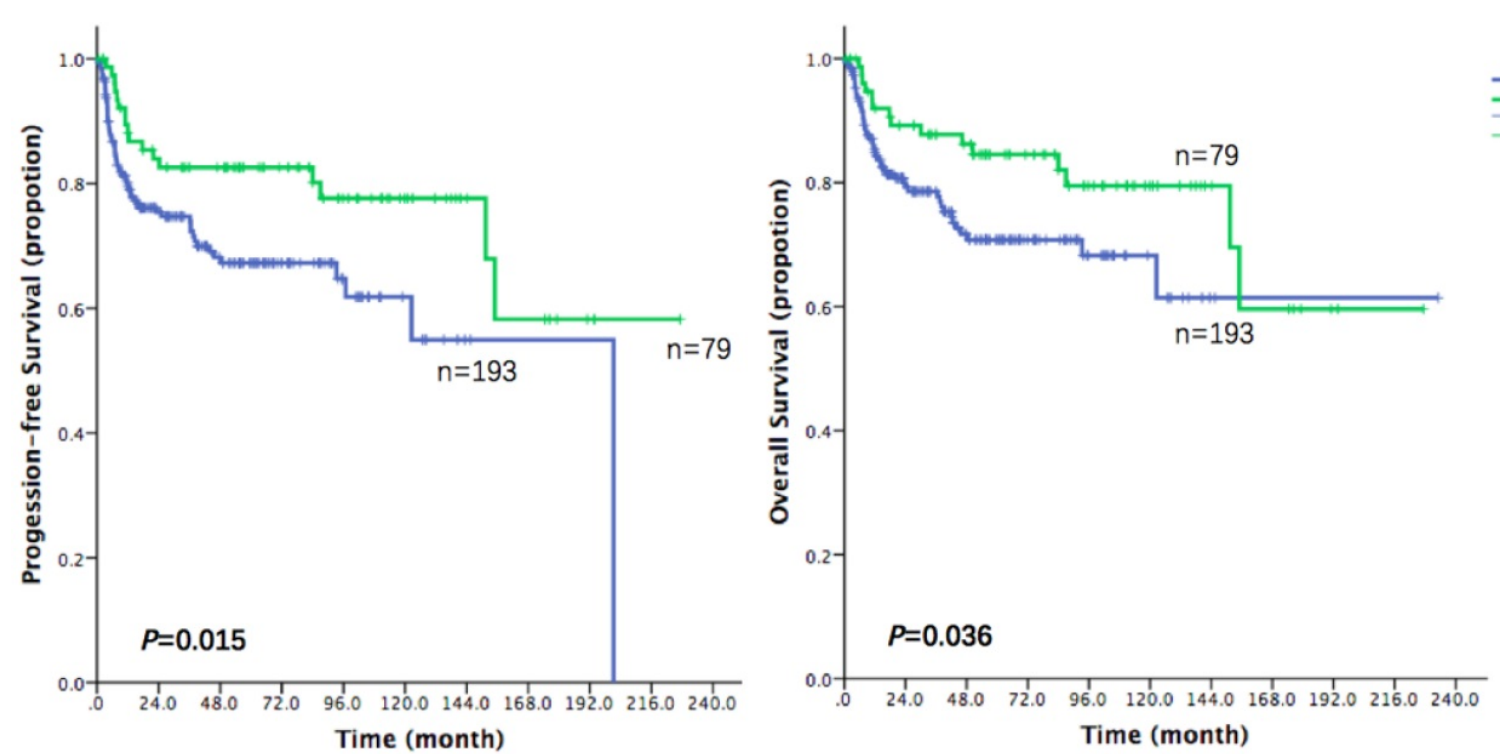

Figure 2. Progression free survival and overall survival depending on whether receiving surgery in 272 patients
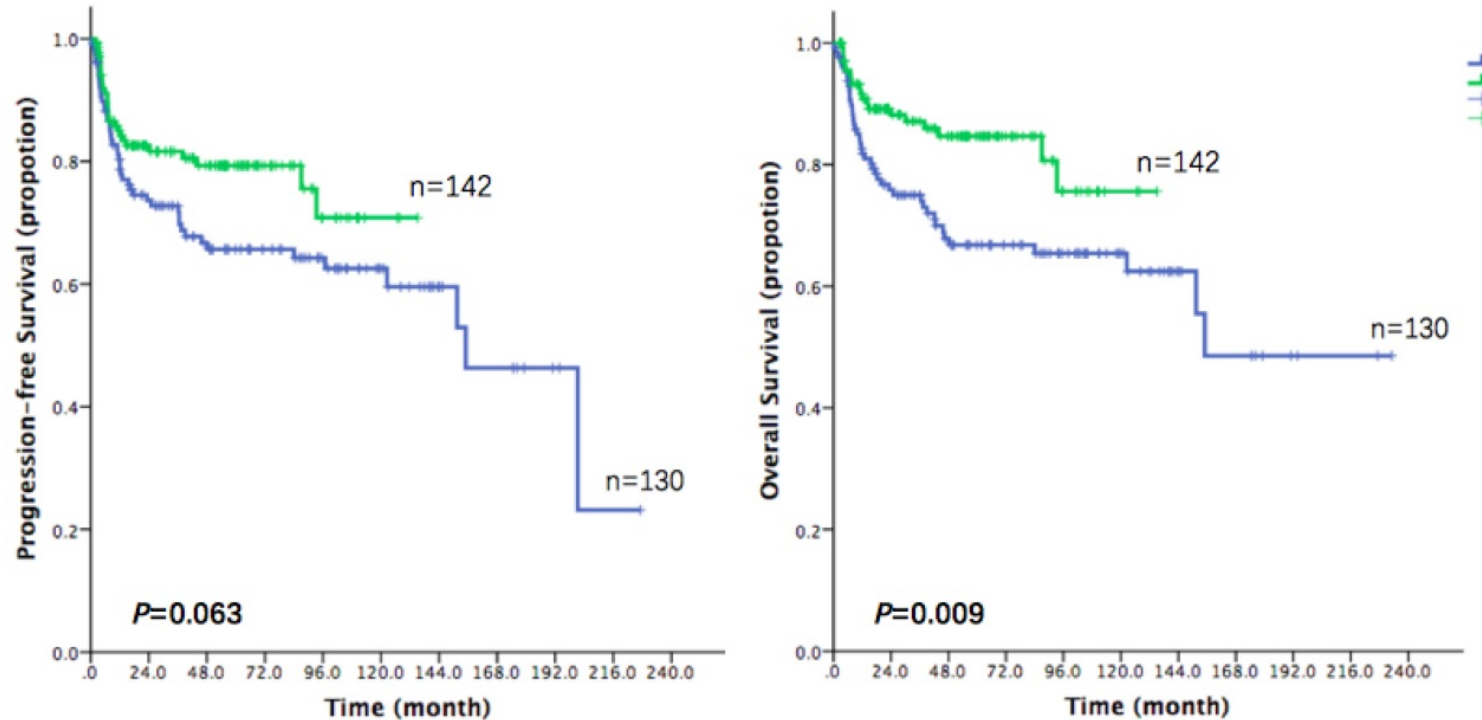

Figure 3. Progression free survival and overall survival depending on whether receiving Rituximab in 272 patients 

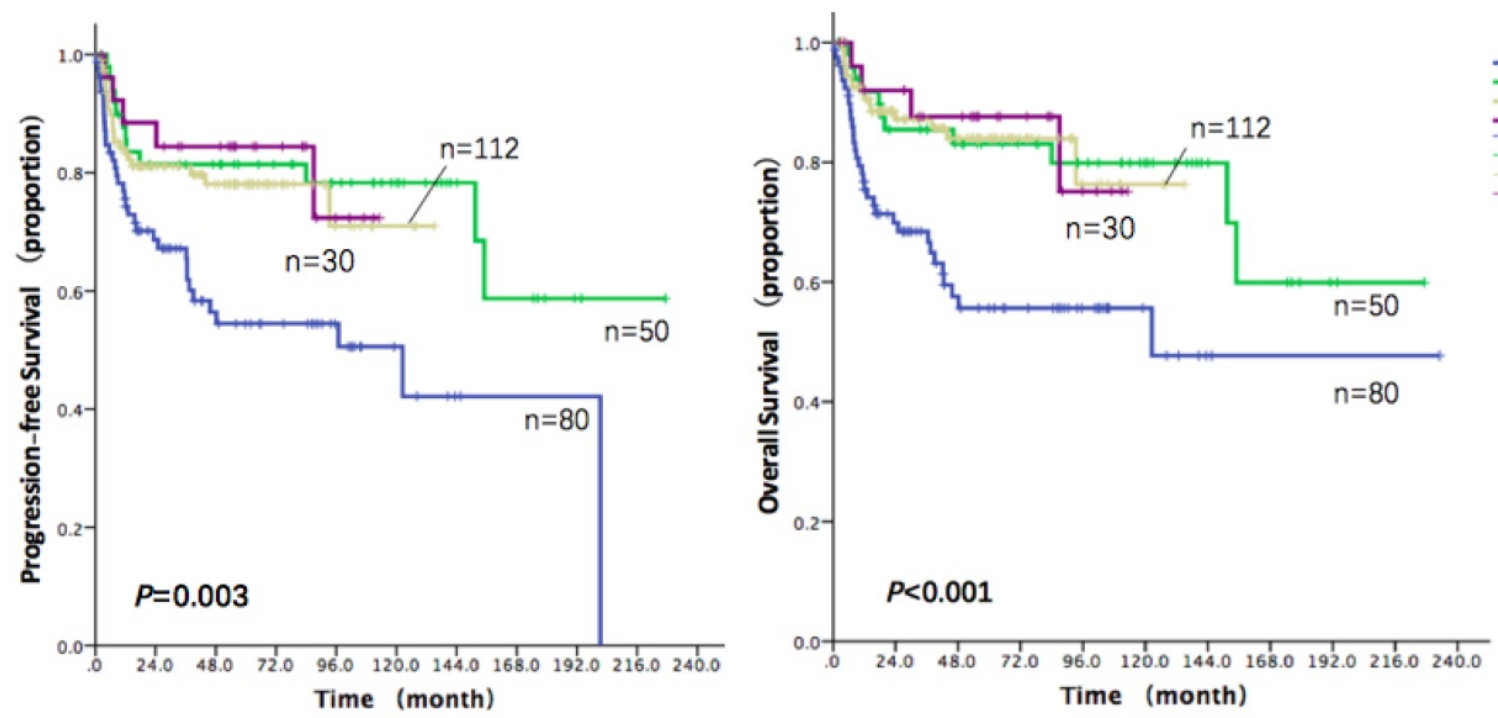

Therapy regimen

7 Croup C Croup $\mathrm{C}+\mathrm{S}$ Croup $R+C$ Croup $R+C+5$ Croup C-censored Croup $\mathrm{R}+\mathrm{C}$-censored Croup $R+C+5$-censored

Figure 4. Progression free survival and overall survival depending on therapy regimens in 272 patients

The similar results were found in patients with different IPI score. In the low risk (IPI 0-2) cohort, neither Rituximab nor surgery showed clinical benefit in PFS ( $p=0.199, p=0.063)$ and OS ( $p=0.091, p=0.056)$. In the high risk (IPI 3-5) cohort, patients received Rituximab had better PFS and OS compared to those who did not receive Rituximab (3-year PFS: $59.2 \%$ vs $34.6 \%, p=0.029$; 3-year OS: $72.9 \%$ vs $34.6 \%, p=0.003$ ). Patients that underwent surgery also had better PFS and OS, albeit the differences were not statistically significant (3-year PFS: $58.2 \%$ vs $46.3 \%, p=0.123$; 3-year OS: $65.5 \%$ vs $53.6 \%, p=0.277)$.

In order to compare the effect of different therapy combinations, patients were further classified into four therapy groups. In the entire cohort, patients received both surgery and Rituximab therapy (Group $\mathrm{R}+\mathrm{C}+\mathrm{S}$ ) failed to show survival benefit compared with those received either surgery (Group $C+S$ ) or Rituximab therapy (Group $\mathrm{R}+\mathrm{C}$ ). Patients receiving only chemotherapy (Group C) had the lowest PFS and OS among the four groups (Group $C$ vs. Group $C+S$ vs. Group R+C vs. Group R+C+S: 3-year PFS: $67.2 \%$ vs. $81.4 \%$ vs. $81.2 \%$ vs. $81.8 \%, p=0.003$; 3 -year OS: $68.4 \%$ vs. $85.4 \%$ vs. $87.2 \%$ vs. $88.6 \%, p<0.001$; Figure 4$)$. The survival of patients in Group $\mathrm{C}+\mathrm{S}$ and Group $\mathrm{R}+\mathrm{C}$ were similar.

\section{Complications affect survival}

Treatment-related complications included gastrointestinal bleeding (21 cases), perforation (4 cases), and obstruction (4 cases), which correlated with poorer outcomes (3-year PFS: $50.9 \%$ vs. $80.2 \%$, $p<0.001$; 3-year OS: $52.5 \%$ vs. $84.7 \%, p<0.001$ ). All these complications were chemotherapy-related. None of surgery-related complications had been observed in the 272 patients.

\section{Prognostic parameters}

Based upon univariate analysis (Table 2), factors that strongly correlated with both PFS and OS included: age, Lugano stage, Eastern Cooperative Oncology Group performance status (ECOG), B symptom, IPI, LDH, Hemoglobin (HGB), ESR, prior gastrointestinal bleeding, therapy regimens and complications $(p<0.05)$. In multivariate analysis, IPI (PFS: $p=0.018$; OS: $p=0.004$ ) and therapy regimens (PFS: $p=0.012$; OS: $p=0.003$ ) were highly predictive for both PFS and OS (Table 3).

\section{Discussion}

According to the statistics of GLOBOCAN 2018 and GLOBOCAN 2012, both new cases and deaths associated with lymphoid neoplasms increased.[12, 13] While approximately $7.5 \%$ of deaths associated with lymphoid neoplasms worldwide occurred in China.[14] This retrospective, multicenter analysis represents the largest sample size so far for PGDLBCL from China. In our study, the 3-year PFS and 3-year OS of the entire cohort were $77.0 \%$ and $81.2 \%$ respectively. IPI and therapy regimens were highly predictive for both PFS and OS. Because of the special primary site, the treatment strategy of PGDLBCL is not the same as nodal DLBCL. Chemotherapy was the core treatment for PGDLBCL. However, the role of surgery, Rituximab, radiotherapy, and the combinations of several therapies above in PGDLBCL therapy remained unclear. We thus evaluated the role of surgery and Rituximab in PGDLBCL and attempted to determine an optimal therapy combination for these patients. 
Table 2. Prognostic factors in univariate analysis

\begin{tabular}{|c|c|c|c|c|c|}
\hline & & 3-year PFS & & 3-yea & \\
\hline & $\mathrm{N}$ & $\%$ & $P$ & $\%$ & $P$ \\
\hline Sex & & & 0.774 & & 0.145 \\
\hline Male & 134 & 75.3 & & 77.2 & \\
\hline Female & 138 & 78.6 & & 85.3 & \\
\hline Age & & & 0.002 & & 0.001 \\
\hline$\leq 60$-year-old & 169 & 80.4 & & 85.1 & \\
\hline$>60$-year-old & 103 & 71.2 & & 74.9 & \\
\hline Lugano Stage & & & $<0.001$ & & $<0.001$ \\
\hline Stage I & 61 & 92.3 & & 95.9 & \\
\hline Stage II & 92 & 89.5 & & 90.4 & \\
\hline Stage IV & 117 & 59.7 & & 66.9 & \\
\hline Unknown & 2 & - & & - & \\
\hline B Symptom & & & $<0.001$ & & $<0.001$ \\
\hline No & 146 & 87.4 & & 91.7 & \\
\hline Yes & 125 & 65.1 & & 69.6 & \\
\hline Unknown & 1 & - & & - & \\
\hline ECOG PS & & & 0.039 & & 0.024 \\
\hline $0-1$ & 255 & 79.2 & & 82.9 & \\
\hline$>1$ & 14 & 38.5 & & 53.8 & \\
\hline Unknown & 3 & - & & - & \\
\hline LDH & & & $<0.001$ & & $<0.001$ \\
\hline Normal $(\leq 240 \mathrm{U} / \mathrm{L})$ & 186 & 88.8 & & 90.9 & \\
\hline Elevated $(>240 \mathrm{U} / \mathrm{L})$ & 78 & 47.9 & & 58.0 & \\
\hline Unknown & 8 & - & & - & \\
\hline IPI & & & $<0.001$ & & $<0.001$ \\
\hline $0-1$ & 146 & 88.2 & & 92.4 & \\
\hline 2 & 55 & 81.0 & & 82.7 & \\
\hline 3 & 43 & 55.7 & & 60.7 & \\
\hline $4-5$ & 21 & 34.3 & & 45.5 & \\
\hline Unknown & 7 & - & & - & \\
\hline HGB & & & $<0.001$ & & $<0.001$ \\
\hline$\geq 120 \mathrm{~g} / \mathrm{L}$ (Male) or $\geq 110 \mathrm{~g} / \mathrm{L}$ (Female) & 140 & 90.0 & & 92.0 & \\
\hline $90 \leq \mathrm{HGB}<120 \mathrm{~g} / \mathrm{L}$ (Male) or $90 \leq \mathrm{HGB}<110 \mathrm{~g} / \mathrm{L}$ (Female) & 79 & 66.7 & & 74.1 & \\
\hline$<90 \mathrm{~g} / \mathrm{L}$ & 53 & 58.3 & & 64.0 & \\
\hline Gastrointestinal bleeding before treatment & & & $<0.001$ & & $<0.001$ \\
\hline No & 150 & 82.8 & & 86.1 & \\
\hline Yes & 69 & 57.3 & & 60.5 & \\
\hline Unknown & 53 & - & & - & \\
\hline ESR & & & 0.004 & & 0.008 \\
\hline $\operatorname{Normal}(\leq 15 \mathrm{~mm} / \mathrm{h})$ & 65 & 88.5 & & 90.0 & \\
\hline Elevated $(>15 \mathrm{~mm} / \mathrm{h})$ & 109 & 68.8 & & 73.4 & \\
\hline Unknown & 98 & - & & - & \\
\hline Rituximab & & & 0.063 & & 0.009 \\
\hline No & 130 & 77.0 & & 79.3 & \\
\hline Yes & 142 & 81.0 & & 87.3 & \\
\hline Surgery & & & 0.015 & & 0.036 \\
\hline No & 193 & 74.7 & & 78.6 & \\
\hline Yes & 79 & 82.6 & & 87.8 & \\
\hline Radiotherapy & & & 0.012 & & 0.178 \\
\hline No & 247 & 79.3 & & 82.9 & \\
\hline Yes & 24 & 56.5 & & 73.4 & \\
\hline Unknown & 1 & - & & - & \\
\hline Therapy regimens & & & 0.003 & & $<0.001$ \\
\hline Chemotherapy & 80 & 67.2 & & 68.4 & \\
\hline Chemotherapy + Surgery & 50 & 81.4 & & 85.4 & \\
\hline Rituximab + Chemotherapy & 112 & 81.2 & & 87.2 & \\
\hline Rituximab + Chemotherapy + Surgery & 30 & 81.8 & & 88.6 & \\
\hline Complication & & & $<0.001$ & & $<0.001$ \\
\hline No & 243 & 80.2 & & 84.7 & \\
\hline Yes & 29 & 50.9 & & 52.5 & \\
\hline
\end{tabular}

ECOG PS: Eastern Cooperative Oncology Group performance status; LDH: Lactate dehydrogenase; IPI: International Prognostic Index; HGB, Hemoglobin; ESR: Erythrocyte sedimentation rate.

Patients that underwent surgery had better outcomes than those who only received pharmaceutical intervention, in both PFS and OS (3-year PFS: $82.6 \%$ vs. $74.7 \%, p=0.015$; 3-year OS: $87.8 \%$ vs. $78.6 \%, p=0.036$ ). Rituximab could improve OS of
PGDLBCL, especially in advanced-stage or high risk (IPI 3-5) patients, while such advantage was not significant in local-stage or low risk (IPI 0-2) cohorts. In previous studies, no difference had been observed between surgery combined with chemotherapy and 
chemotherapy alone.[15-18] The value of Rituximab remained controversial. Some of studies advocated for Rituximab in PGDLBCL,[19-22] while others held opposing views.[18, 23] All these studies above discussed the value of either surgery or Rituximab in PGDLBCL. While, our study for the first time compared all four commonly adopted regimens: chemotherapy alone, chemotherapy + surgery, chemotherapy + Rituximab, and chemotherapy + surgery + Rituximab. We found that the survival of patients in Group $\mathrm{C}+\mathrm{S}$ and Group $\mathrm{R}+\mathrm{C}$ were similar (3-year PFS: $81.4 \%$ vs. $81.2 \%$, $p=0.551$; 3-year OS: $85.4 \%$ vs. $87.2 \%, p=0.948)$, while patients received both surgery and Rituximab therapy (Group $\mathrm{R}+\mathrm{C}+\mathrm{S}$ ) failed to show survival benefit compared to those who underwent either surgery (Group $\mathrm{C}+\mathrm{S}$ ) or Rituximab therapy (Group $\mathrm{R}+\mathrm{C}$ ). These results suggested that chemotherapy alone was not enough for PGDLBCL, and the clinical outcome could not be further improved by adding either rituximab or surgery to the two combinations. Although the effect of surgery and Rituximab were similar. The better therapy choice was varied from different patients. Although we did not observe any post-surgery complications, Koch $\mathrm{P}$ reported that surgery increased the risk of bleeding, perforation, and obstruction in perioperative period, leading to postpone of chemotherapy.[24] Furthermore, late complications of surgery, such as malabsorption syndrome, marginal ulcer, and alkaline reflux gastritis, may emerge several years later, which could cause severe nutrition depletion and reduction in the quality of life. In light of these, our team is inclined to choose chemotherapy combined with Rituximab as the first-line therapy for most of PGDLBCL. However, according to our data, treatment-related complications correlated with poorer outcomes (3-year PFS: $50.9 \%$ vs. $80.2 \%$, $p<0.001$; 3-year OS: $52.5 \%$ vs. $84.7 \%, p<0.001$ ). If we predicted that the patients had high risk of bleeding or perforation after receiving pharmaceutical intervention, receiving surgery before chemotherapy maybe reduce treatment-related complications and improve survival. In that case, chemotherapy combined with surgery could be an optimal chooses.

In addition, we provided a little evidence to reveal the role of radiotherapy in PGDLBCL. In our study, all the 24 patients receiving radiotherapy during initial treatment had at least one of the following two situations: 1) existing residual lesion after chemotherapy \pm surgery; 2) intolerance to chemotherapy. As a result, the inclusion of radiotherapy failed to show the improvement of survival. Besides using radiotherapy as salvage treatment, three cycles of $\mathrm{CHOP}$ followed by involved field radiotherapy was used effectively and well-tolerated in localized PGDLBCL patients.[25, 26] However, in Rituximab era, it remains unclear whether Rituximab can replace radiotherapy as a better choice for local-stage PGDLBCL. For local-stage patients, who achieved complete remission after four cycles of chemotherapy, consolidation radiotherapy could improve local control rate.[27] However, this regimen did not lead to any improvement in PFS and OS. Furthermore, Japanese researchers reported the correlation between PGDLBCL treated by radiotherapy \pm chemotherapy and gastric adenocarcinoma.[28] Randomized controlled trial was strongly needed to evaluate the necessity and safety of radiotherapy in PGDLBCL.

Table 3. Prognostic factors in multivariate analysis

\begin{tabular}{|c|c|c|c|c|c|c|}
\hline & PFS & & & OS & & \\
\hline & HR & $95 \% \mathrm{CI}$ & $p$ & HR & $95 \% \mathrm{CI}$ & $p$ \\
\hline B symptom & 0.527 & $0.252-1.101$ & 0.088 & 0.487 & $0.219-1.084$ & 0.078 \\
\hline IPI & & & 0.018 & & & 0.004 \\
\hline $0-1$ vs. $4-5$ & 0.245 & $0.087-0.695$ & 0.008 & 0.163 & $0.015-0.522$ & 0.002 \\
\hline 2 vs. $4-5$ & 0.284 & $0.099-0.810$ & 0.019 & 0.274 & $0.090-0.836$ & 0.023 \\
\hline 3 vs. $4-5$ & 0.722 & $0.291-1.793$ & 0.483 & 0.782 & $0.298-2.057$ & 0.619 \\
\hline HGB & & & 0.345 & & & 0.258 \\
\hline $\begin{array}{l}\text { Male: } \geq 120 \mathrm{~g} / \mathrm{L} \text { vs. }<90 \mathrm{~g} / \mathrm{L} \\
\text { Female: } \geq 110 \mathrm{~g} / \mathrm{L} \text { vs. }<90 \mathrm{~g} / \mathrm{L}\end{array}$ & 1.065 & $0.439-2.587$ & 0.889 & 1.752 & $0.655-4.696$ & 0.264 \\
\hline $\begin{array}{l}\text { Male: } 90 \leq \mathrm{HGB}<120 \mathrm{~g} / \mathrm{L} \text { vs. }<90 \mathrm{~g} / \mathrm{L} \\
\text { Female: } 90 \leq \mathrm{HGB}<110 \mathrm{~g} / \mathrm{L} \text { vs. }<90 \mathrm{~g} / \mathrm{L}\end{array}$ & 1.653 & $0.767-3.565$ & 0.199 & 2.041 & $0.868-4.801$ & 0.102 \\
\hline ESR & 0.774 & $0.346-1.73$ & 0.533 & 0.849 & $0.360-2.000$ & 0.708 \\
\hline Therapy regimens & & & 0.012 & & & 0.003 \\
\hline C vs. $\mathrm{R}+\mathrm{C}+\mathrm{S}$ & 4.129 & $1.226-13.909$ & 0.022 & 4.584 & $1.323-15.879$ & 0.016 \\
\hline $\mathrm{C}+\mathrm{S}$ vs. $\mathrm{R}+\mathrm{C}+\mathrm{S}$ & 1.876 & $0.463-7.597$ & 0.378 & 2.187 & $0.530-9.019$ & 0.279 \\
\hline $\mathrm{R}+\mathrm{C}$ vs. $\mathrm{R}+\mathrm{C}+\mathrm{S}$ & 1.582 & $0.462-5.419$ & 0.465 & 1.221 & $0.339-4.403$ & 0.760 \\
\hline Complication & 0.553 & $0.261-1.172$ & 0.122 & 0.480 & $0.224-1.032$ & 0.060 \\
\hline
\end{tabular}

PFS: progression free survival; OS: overall survival; HR: Hazard risk; 95\% CI: 95\% confidence interval; IPI: International Prognostic Index; HGB, Hemoglobin; ESR:

Erythrocyte sedimentation rate; C: Chemotherapy; C+S: Chemotherapy + Surgery; R+C: Rituximab + Chemotherapy; R+C+S: Rituximab + Chemotherapy + Surgery. 
Furthermore, immunotherapy has risen to the fifth main therapeutic modality for DLBCL, following chemotherapy, targeted therapy, surgery, and radiotherapy. Monoclonal antibodies targeting programmed death-1 (PD-1), programmed death-ligand 1 (PD-L1) and cytotoxic T-lymphocyte antigen-4 (CTLA-4) had been used alone or in combination with anti-CD20 antibody in a subset of DLBCL.[29] FDA had also approved two chimeric antigen receptor (CAR) $T$ products for relapse and refractory B cell lymphoma. Future administration of immunotherapy regimens will be more dependent upon patients' biomarkers, rather than on histological types and subtypes.[30] As a special type of DLBCL, biomarkers unique to PGDLBCL should be actively explored, which may facilitate the discovery of safer and more effective therapeutics for this disease.

In summary, our results suggested that the combinations of chemotherapy and surgery, or chemotherapy and Rituximab, can raise the survival rate of PGDLBCL patients, compared to chemotherapy alone. However, the clinical outcome could not be further improved by adding either Rituximab or surgery to the two combinations. Rituximab showed clinical benefit in survival, especially in advanced-stage or high risk (IPI 3-5) patients. The inclusion of radiotherapy during initial treatment did not improve survival in our study. IPI and therapy regimens are independent predictors of outcomes. The future prospective trial is warranted.

\section{Acknowledgements}

Jun Zhu, Xinan Cen, and Haiyan Yang designed the research; Meng Wu and Jun Zhu wrote the article; and all authors provided study materials or patients and approved the article. We would like to thank Medpion (Beijing) Medical Technology Co., Ltd. for their help in data collection and statistical analysis.

\section{Funding}

This study was funded by the National Natural Science Foundation of China (No. 81870154, 81470368, 81670187, 81600164, 81700197), the Beijing Natural Science Foundation (No. 7172047, 7172046), Capital's Funds for Health Improvement and Research (No. 2018-1-2151), the Beijing Municipal Administration of Hospitals' Ascent Plan (No. DFL20151001), the Beijing Medical and Health Foundation (No.2221-16-157), the key topics fund of Beijing Municipal Science and Technology Commission (No. Z141107002514017), Zhejiang medical and health science project (No. 2015KYB067), the key funding program of Zhejiang medical and health platform (No. 2015ZDA021).

\section{Competing Interests}

The authors have declared that no competing interest exists.

\section{References}

1. Al-Hamadani M, Habermann TM, Cerhan JR, Macon WR, Maurer MJ, Go RS. Non-Hodgkin lymphoma subtype distribution, geodemographic patterns, and survival in the US: A longitudinal analysis of the National Cancer Data Base from 1998 to 2011. Am J Hematol. 2015; 90: 790-5.

2. Castillo JJ, Winer ES, Olszewski AJ. Sites of extranodal involvement are prognostic in patients with diffuse large B-cell lymphoma in the Rituximab era: an analysis of the Surveillance, Epidemiology and End Results database. Am J Hematol. 2014; 89: 310-4.

3. Ferrucci PF, Zucca E. Primary gastric lymphoma pathogenesis and treatment: what has changed over the past 10 years? Br J Haematol. 2007; 136: 521-38.

4. Aisenberg AC. Coherent view of non-Hodgkin's lymphoma. J Clin Oncol. 1995; 13: 2656

5. Kuo SH, Cheng AL. Helicobacter pylori and mucosa-associated lymphoid tissue: what's new. Hematology. 2013; 2013: 109.

6. Howell JM, Auergrzesiak I, Zhang J, Andrews CN, Stewart D, Urbanski SJ. Increasing incidence rates, distribution and histological characteristics of primary gastrointestinal non-Hodgkin lymphoma in a North American population. Can J Gastroenterol. 2012; 26: 452-6.

7. Ding W, Zhao S, Wang J, Yang Q, Sun H, Yan J, et al. Gastrointestinal Lymphoma in Southwest China: Subtype Distribution of 1,010 Cases Using the WHO (2008) Classification in a Single Institution. Acta Haematol. 2016; 135: 21-8.

8. Nakamura S, Matsumoto T, Iida M, Yao T, Tsuneyoshi M. Primary gastrointestinal lymphoma in Japan: a clinicopathologic analysis of 455 patients with special reference to its time trends. Cancer. 2003; 97: 2462-73.

9. Swerdlow SH, Campo E, Harris NL, Jaffe ES, Pileri SA, Stein H, Thiele J, Vardiman JW. WHO Classification of Tumours of Haematopoietic and Lymphoid Tissues. 2008.

10. Rohatiner A, D'Amore OBOF, Coiffier B, Crowther D, Gospodarowicz M, Isaacson $\mathrm{P}$, et al. Report on a workshop convened to discuss the pathological and staging classifications of gastrointestinal tract lymphoma. Ann Oncol. 1994; 5: 397.

11. Cheson BD, Horning SJ, Coiffier B, Shipp MA, Fisher RI, Connors JM, et al. Report of an international workshop to standardize response criteria for non-Hodgkin's lymphomas. NCI Sponsored International Working Group. J Clin Oncol. 1999; 17: 1244.

12. Bray F, Ferlay J, Soerjomataram I, Siegel RL, Torre LA, Jemal A. Global cancer statistics 2018: GLOBOCAN estimates of incidence and mortality worldwide for 36 cancers in 185 countries. CA Cancer J Clin. 2018; 68: 394-424.

13. Torre LA, Bray F, Siegel RL, Ferlay J, Lortet-Tieulent J, Jemal A. Global cancer statistics, 2012. CA Cancer J Clin. 2015; 65: 87-108.

14. Liu W, Liu J, Song Y, Wang X, Zhou M, Wang L, et al. Mortality of lymphoma and myeloma in China, 2004-2017: an observational study. J Hematol Oncol. 2019; 12: 22.

15. Willich NA, Reinartz G, Hiddemann W, Tiemann M, Kocik J, Koch P. Operative and conservative management of primary gastrointestinal lymphoma: Interim results of a German multicenter study. Int J Radiat Oncol Biol Phys. 2000; 46: 895-901.

16. Avilés A, Nambo MJ, Neri N, Huerta-Guzmán J, Cuadra I, Alvarado I, et al. The role of surgery in primary gastric lymphoma: results of a controlled clinical trial. Ann Surg. 2004; 240: 44-50.

17. M B, A R-F, E L, C H, A D, P A, et al. Surgical resection plus chemotherapy versus chemotherapy alone: comparison of two strategies to treat diffuse large B-cell gastric lymphoma. Ann Oncol. 2003; 14: 1751-7.

18. Mehmet $\mathrm{K}$, Sener $\mathrm{C}$, Uyeturk $\mathrm{U}$, Seker M, Tastekin $\mathrm{D}$, Tonyali $\mathrm{O}$, et al Treatment modalities in primary gastric lymphoma: the effect of Rituximab and surgical treatment. A study by the Anatolian Society of Medical Oncology. Contemp Oncol (Pozn). 2014; 18: 273-8.

19. Avilés A, Castañeda C, Cleto S, Neri N, Huerta-Guzmán J, Gonzalez M, et al. Rituximab and chemotherapy in primary gastric lymphoma. Cancer Biother Radiopharm. 2009; 24: 25-8.

20. Leopardo D, Lorenzo GD, Renzo AD, Federico P, Luponio S, Buonerba C, et al. Efficacy of Rituximab in gastric diffuse large B cell lymphoma patients. World J Gastroenterol. 2010; 16: 2526.

21. Zhang J, Li G, Yang H, Liu X, Cao J. Rituximab in treatment of primary gastric diffuse large B-cell lymphoma. Leuk Lymphoma. 2012; 53: 2175-81.

22. Chihara D, Oki Y, Ine S, Kato H, Onoda H, Taji H, et al. Primary gastric diffuse large B-cell Lymphoma (DLBCL): analyses of prognostic factors and value of pretreatment FDG-PET scan. Eur J Haematol. 2010; 84: 493-8.

23. Sohn BS, Kim SM, Yoon DH, Kim S, Lee DH, Kim JH, et al. The comparison between $\mathrm{CHOP}$ and $\mathrm{R}-\mathrm{CHOP}$ in primary gastric diffuse large $\mathrm{B}$ cell lymphoma. Ann Hematol. 2012; 91: 1731.

24. Koch P, Probst A, Berdel WE, Willich NA, Reinartz G, Brockmann J, et al. Treatment results in localized primary gastric lymphoma: Data of patients registered within the German Multicenter Study (GIT NHL 02/96). J Clin Oncol. 2005; 23: 7050-9. 
25. Park YH, Lee SH, Kim WS, Bang SM, Ryoo BY, Yang SH, et al. CHOP followed by involved field radiotherapy for localized primary gastric diffuse large B-cell lymphoma: Results of a multi center phase II study and quality of life evaluation. Leuk Lymphoma. 2006; 47: 1253.

26. Ishikura S, Tobinai K, Ohtsu A, Nakamura S, Yoshino T, Oda I, et al. Japanese multicenter phase II study of CHOP followed by radiotherapy in stage I-II, diffuse large B-cell lymphoma of the stomach. Cancer Sci. 2005; 96: 349-52.

27. Li Q, Li W, Wang L, Wang W, Niu S, Bi X, et al. Consolidation Radiotherapy in Stage IE- IIE, Non-Bulky Primary Gastric Diffuse Large B-Cell Lymphoma with Post-Chemotherapy Complete Remission. PLoS One. 2015; 10: e0133469.

28. Inaba K, Kushima R, Murakami N, Kuroda Y, Harada K, Kitaguchi M, et al. Increased risk of gastric adenocarcinoma after treatment of primary gastric diffuse large B-cell lymphoma. BMC Cancer. 2013; 13: 499.

29. Ok CY, Young $\mathrm{KH}$. Checkpoint inhibitors in hematological malignancies. J Hematol Oncol. 2017; 10: 103.

30. Li Z, Song W, Rubinstein M, Liu D. Recent updates in cancer immunotherapy: a comprehensive review and perspective of the 2018 China Cancer Immunotherapy Workshop in Beijing. J Hematol Oncol. 2018; 11: 142. 